\title{
Beyond the limits of conventional Stark deceleration
}

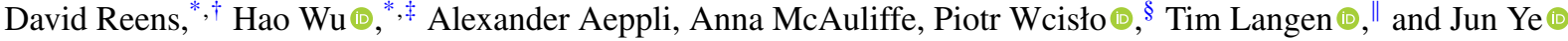 \\ JILA, National Institute of Standards and Technology and the University of Colorado \\ and Department of Physics, University of Colorado, Boulder, Colorado 80309-0440, USA
}

(Received 22 March 2020; accepted 22 June 2020; published 17 July 2020)

\begin{abstract}
Stark deceleration enables the production of cold and dense molecular beams with applications in trapping, collisional studies, and precision measurement. Improving the efficiency of Stark deceleration, and hence the achievable molecular densities, is central to unlock the full potential of such studies. One of the chief limitations arises from the transverse focusing properties of Stark decelerators. We introduce an operation strategy that circumvents this limit without any hardware modifications, and experimentally verify our results for hydroxyl radicals. Notably, improved focusing results in significant gains in molecule yield with increased operating voltage, formerly limited by transverse-longitudinal coupling. At final velocities sufficiently small for trapping, molecule flux improves by a factor of 4 , and potentially more with increased voltage. The improvement is more significant for less readily polarized species, thereby expanding the class of candidate molecules for Stark deceleration.
\end{abstract}

DOI: 10.1103/PhysRevResearch.2.033095

\section{INTRODUCTION}

Over the past two decades, Stark deceleration [1,2], where time-varying inhomogeneous electric fields are used to slow polarizable molecules, has enabled groundbreaking collisional [3-5] and spectroscopic [6-9] studies of a variety of species. Subsequent trap loading $[10,11]$ greatly enhances interrogation time for such studies [12] and opens the door for further manipulation [13]. Alongside the history of achievements enabled by Stark deceleration runs a parallel ongoing saga surrounding their efficient operation. Many important steps have been made, not only in understanding the flaws of the canonical pulsed decelerator [14,15], but also in addressing them through the use of overtones [16,17], extra switching [18], or mixed phase angles [19,20]. Even with these advances, outstanding inefficiencies of the pulsed decelerator, particularly with regard to transverse phase stability,

\footnotetext{
*These authors contributed equally to this work.

${ }^{\dagger}$ Present address: Lincoln Laboratory, Massachusetts Institute of Technology, Lexington, Massachusetts 02420, USA; dave.reens@colorado.edu

${ }^{\ddagger}$ Present address: Department of Physics and Astronomy, University of California, Los Angeles, California 90095, USA; hao.wu@colorado.edu

${ }^{\S}$ Present address: Institute of Physics, Faculty of Physics, Astronomy and Informatics, Nicolaus Copernicus University, Grudziadzka 5, PL-87-100 Toruń, Poland.

"Present address: 5. Physikalisches Institut and Center for Integrated Quantum Science and Technology (IQST), Universität Stuttgart, Pfaffenwaldring 57, 70569 Stuttgart, Germany.

Published by the American Physical Society under the terms of the Creative Commons Attribution 4.0 International license. Further distribution of this work must maintain attribution to the author(s) and the published article's title, journal citation, and DOI.
}

have motivated alternative geometries such as interspersed quadrupole focusing [15] and traveling wave deceleration [21-23]. Although traveling wave deceleration takes a strong step toward truly efficient operation, it comes with significant engineering challenges. These may be partially addressed by the combined use of pulsed and traveling wave devices [24], or using traveling wave geometry with pulsed electronics $[25,26]$. In Zeeman deceleration, the magnetic analog of Stark deceleration, early demonstrations [27,28] were later improved through the use of anti-Helmholtz configurations with better transverse focusing properties [29,30]. Lacking a comparable breakthrough for Stark devices, others have resorted to brand new geometries [31], or combined the Stark and Zeeman approaches in a single device [32,33].

In contrast, we present a strategy for Stark decelerators that works with conventional geometry and electronics. Our strategy fully resolves transverse challenges and improves yields at all final speeds. It is readily applicable to existing decelerators and thus promises improvements in fields ranging from collisional studies and molecular trapping to precision measurements [34].

\section{CONVENTIONAL OPERATION}

To understand this strategy, we revisit the operating principles of a Stark decelerator. The conventional pulsed Stark decelerator consists of an electrode array with alternating pairs of pins orthogonal to a beam line that passes between them [see Fig. 1, top right, for a three-dimensional (3D) render]. In the conventional $S=1$ strategy [2], as low-field seeking molecules [35] approach a charged pin pair, they are polarized by the strong electric field and exchange kinetic energy for internal potential energy, effectively climbing a potential hill. The strong field is then abruptly removed by high-voltage switches before the molecules have a chance to regain kinetic energy (Fig. 1, bottom row). It is customary to 

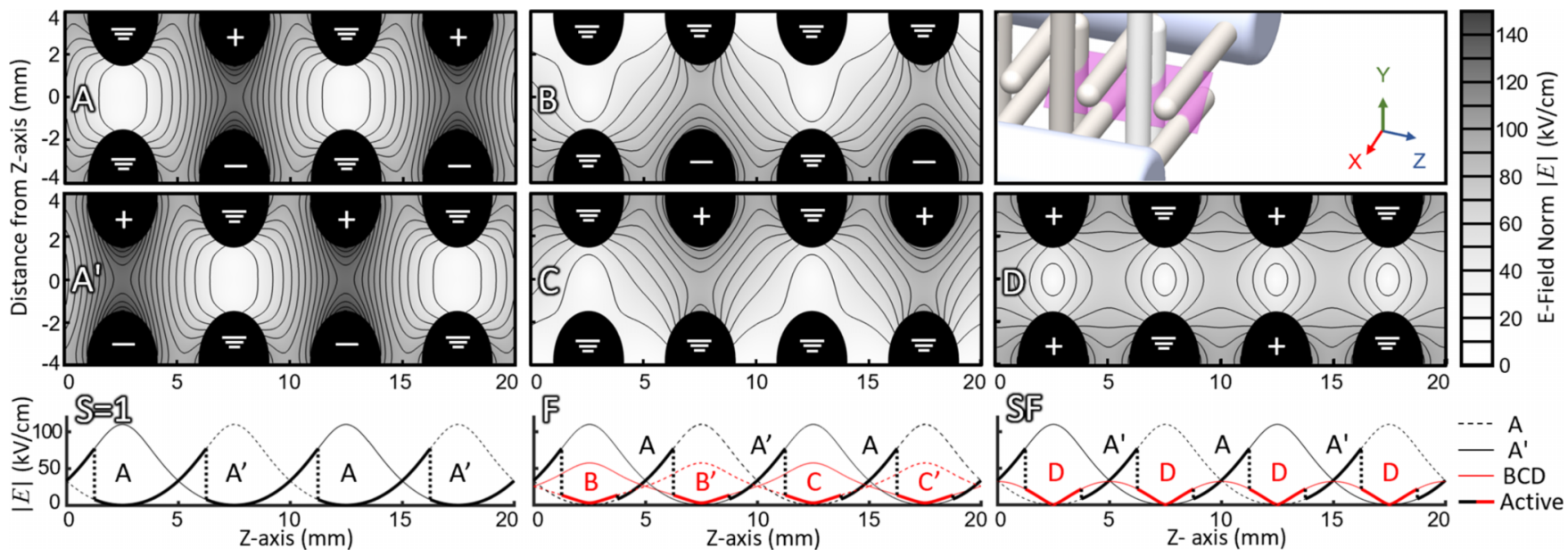

FIG. 1. Beyond the limits: conventional Stark deceleration (left column) and transversely focusing variants (middle and right columns), Conventional Stark deceleration utilizes a single distribution of electric field (A, top) and its translation plus $90^{\circ}$ rotation $\left(\mathrm{A}^{\prime}\right.$, middle) in an alternating fashion termed " $\mathrm{S}=1$ " operation (bottom, aligned) [16]. The bottom panel describes $\mathrm{S}=1$ by plotting the potential experienced by an ideal "synchronous molecule" as it propagates exactly down the center axis of the decelerator (bold). Abrupt changes (bold dashed lines) in the potential the synchronous molecule experiences are achieved by rapidly switching between A and A' (labels indicate where each is active) via fast high-voltage switches. Distribution A scarcely focuses, and is not active where it most strongly focuses $(z=7.5 \mathrm{~mm}$, note increasing field strength of axis). Distributions are shown in the diagonally slicing plane visible in the 3D render (top right, pink). A new focusing mode (F, middle column) circumvents this focusing limitation through the incorporation of new distributions B and C (and their primes, not shown, which relate as do A and $\mathrm{A}^{\prime}$ ). These distributions do not focus on their own but only when averaged together. A and $\mathrm{A}^{\prime}$ are still used close to 5 and $10 \mathrm{~mm}$ as in $\mathrm{S}=1$, with the result that the energy removed per pin pair (total length of bold dashed lines) is equivalent to $\mathrm{S}=1$. Mode $\mathrm{SF}$ (strong focusing, right column) simply replaces B, C, and their primes with $\mathrm{D}$, which is more strongly focusing but challenging to implement experimentally.

discuss the behavior of an idealized "synchronous molecule" that travels along the decelerator axis with zero transverse velocity. The switching is timed so that the synchronous molecule loses some fixed energy per switch. It is essential that the synchronous molecule travel only partway up each hill, so that molecules that are ahead of the synchronous molecule get more energy removed, and vice versa. This generates a longitudinal restoring force for the ensemble, centered on the synchronous molecule. Transversely, restoring force is not inherited from switching events but arises from the focusing properties of the electric field distributions that the electrode array generates (Fig. 1, top and middle rows). When molecules reside in a region where the electric field is stronger off axis than on, they experience transverse focusing. Although transverse focusing varies rapidly with longitudinal coordinate in the decelerator, these variations are too fast for molecules to follow. We may therefore make a high-speed approximation, and time-average transverse and longitudinal forces to obtain a "traveling trap" for the molecules [36], which translates along the device and decelerates according to a ramp of the switching frequency. This is valid provided that $v_{z} / D \gg f$, where $v_{z}$ is the longitudinal velocity of the molecules, $D$ the distance between pin pairs, and $f$ the oscillation frequency in the traveling trap.

Conventionally, pins are always charged in bipolar pairs, in which case transverse focusing occurs between the charged pin pair, but not significantly elsewhere (Fig. 1, A). Molecules do not regularly access the focusing region, since pins are grounded before the synchronous molecule reaches them as discussed above. As the molecules pass between grounded pins, the transverse field is actually slightly defocusing [37].
Their transverse confinement also varies with how strongly the molecules are decelerated, and with their distance from the synchronous molecule along the decelerator axis. Such a dependence of transverse confinement on longitudinal position is known as transverse-longitudinal coupling, and it gives rise to the situation that molecules which are coldest longitudinally are less well confined transversely [14]. The use of deceleration overtones such as $\mathrm{S}=3$ [16] alleviates coupling by allowing molecules to fully transit between charged pin pairs regardless of their relative position with the synchronous molecule. This mode of operation leverages the full focusing properties of the conventional field distribution, but at the expense of only using $1 / 3$ of the pin pairs for removing energy.

\section{FOCUSING OPERATION}

Our strategy is to introduce new field distributions with strong transverse restoring forces when the synchronous molecule is between grounded pin pairs (see the Appendix), but to retain the use of the conventional distribution otherwise. Field distributions that focus between grounded pins can be created by charging the neighboring pins to voltages that do not sum to zero. Field lines then extend toward the grounded pin pair, creating a focusing two-dimensional (2D) quadrupole structure. Possibilities include charging only a single pin as in Fig. 1 ( $\mathrm{B}$ and $\mathrm{C}$ ), or charging both to the same voltage (Fig. 1, D). We name the operating modes employing these distributions focusing (F) and strong focusing (SF). We restrict attention to distributions that make use of the same 
(a)

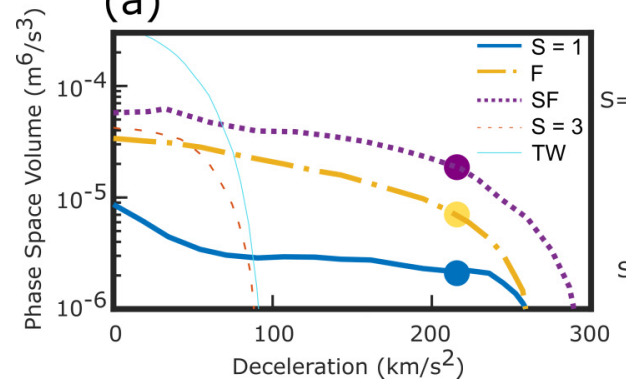

(b)

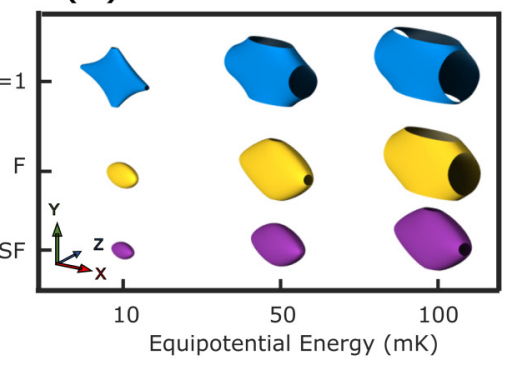

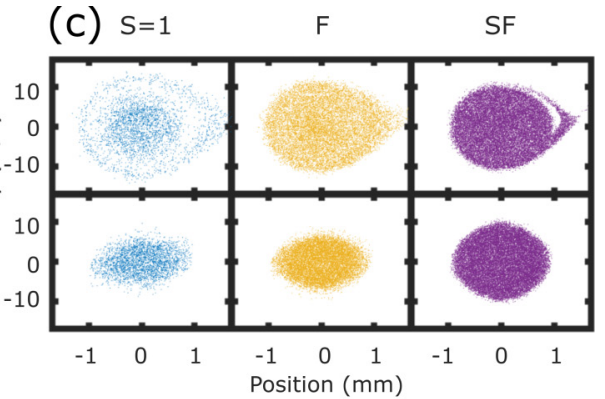

FIG. 2. Simulation results of different deceleration modes. (a) Simulated phase-space volume captured by different modes of operation, for varying decelerations and elapsed time fixed at $3 \mathrm{~ms}$. A 10-kV peak-to-peak traveling wave (TW) deceleration and $\pm 12.5 \mathrm{kV} \mathrm{S}=3$ are also plotted for comparison. Three solid dots correspond to the deceleration used in (b) and (c), about $200 \mathrm{~km} / \mathrm{s}^{2}$. (b) Equipotentials of the traveling trap generated for three modes. Lack of closure of an equipotential indicates the possibility of molecule escape. The $z$ axis corresponds to the longitudinal direction. (c) Phase-space fillings, both longitudinal (top) and transverse (bottom), for the labeled operation modes after 3 ms of travel. The surviving number of molecules is $3.0 \times 10^{3}, 1.1 \times 10^{4}$, and $2.4 \times 10^{4}$, respectively. Note dramatic improvements in homogeneity and flux, without significant broadening to larger velocity classes.

triplet of voltages $(0, \pm 12.5 \mathrm{kV}$ for our device) that are applied conventionally, but rearranged.

In order to best compare these modes in a deviceindependent way, we perform simulations of hydroxyl radicals $(\mathrm{OH})$ with fixed travel time $(3 \mathrm{~ms})$ and varying deceleration rate [see Fig. 2(a)]. Fixing travel time facilitates comparison between long devices with fast initial speeds and short devices with slower initial speeds, for example. $\mathrm{S}=1$ delivers the smallest phase-space volumes, although it provides at least some flux even at high deceleration. Remarkably, the F mode offers comparable phase-space volume to $\mathrm{S}=3$, but with triple the deceleration. The SF mode makes more dramatic improvements, extending significant gains to even higher decelerations than possible with any other studied modes. For the traveling wave (TW) decelerator comparison in Fig. 2(a), 10-kV sine waves are assumed, to our knowledge the largest used to decelerate molecules to rest [24]. The TW mode offers a good phase-space volume but is limited to a smaller maximum deceleration, similar to $\mathrm{S}=3$. All modes besides TW use the rather small $2 \times 2 \mathrm{~mm}^{2}$ open area of our device, while TW devices use rings of 4-mm inner diameter. If these modes are used with a $3 \times 3 \mathrm{~mm}^{2}$ [17] or a $4 \times 4 \mathrm{~mm}^{2}$ [38] device, phase-space volume compares more favorably with the TW mode. Unlike the TW mode, however, the performance of $\mathrm{F}, \mathrm{SF}$, and $\mathrm{S}=1$ all degrade significantly when $v_{z}<50 \mathrm{~m} / \mathrm{s}$ and the high-speed approximation breaks down.

In understanding the mechanism for this improved performance, it is helpful to visually inspect the traveling trap generated by each mode [see Fig. 2(b)]. Here we plot equipotential surfaces for these traps at three different energies and for $200 \mathrm{~km} / \mathrm{s}^{2}$ deceleration. The openings in these surfaces occur when the surface reaches the $2 \times 2 \mathrm{~mm}^{2}$ transverse limits of our decelerator geometry. Molecules reaching this boundary are lost. Molecules may also be lost longitudinally, often remaining transversely focused but no longer decelerating with the synchronous molecule. For the $S=1$ mode, the $10 \mathrm{mK}$ equipotential is transversely broad and even contains four small openings. This corresponds to the transverse-longitudinal coupling problem discussed above. The improvements in operation efficiency for F and SF modes correspond to improved tightness and closure as evident in all equipotentials shown.

In Fig. 2(c), the longitudinal and transverse phase-space fillings are compared for all modes, with $200 \mathrm{~km} / \mathrm{s}^{2}$ deceleration and $3 \mathrm{~ms}$ travel time as before. All modes are initialized with the same homogeneous phase-space density (PSD). This is valid when the initial beam source generates a much broader distribution than the volume accepted by the traveling trap. In the longitudinal direction, most supersonic expansions satisfy this, with the exception of those performed with a helium buffer gas, which can reach temperatures as low as $40 \mathrm{mK}$ expanding from room temperature [39]. As can be seen, the distribution is nearly homogeneous after deceleration for all modes except $S=1$. Increases in point density from $S=1$ to $\mathrm{F}$ and to SF arise from increases in the phase-space volume captured by those operating modes, which is then projected onto the planes shown. Phase-space density is not enhanced, nor could it be by the reversible, nondissipative Stark deceleration technique. However, preparing an optimally shaped distribution minimizes subsequent losses in phase-space density arising from potentially poor mode matching. For example, a trap with an acceptance comparable to the outer dimensions of the $S=1$ mode will be underfilled by the $S=1$ mode due to the prominent missing ring, while the F mode will not do this, effectively quadrupling the phase-space density loaded in such a trap. Most realistic traps possess comparable transverse and longitudinal phase-space acceptances due to ergodicity and cross-dimensional couplings. The SF mode is appealing in this respect with nearly identical transverse and longitudinal acceptance.

\section{EXPERIMENTAL VALIDATION}

We experimentally measure the performance of $\mathrm{F}$ and $\mathrm{S}=1$ for $\mathrm{OH}$ (see Fig. 3). If the distributions shown in Fig. 1 (A-C) are properly arranged, the F mode may be implemented from $S=1$ simply by turning off one pin in a pair earlier than the other, and cycling which pin is chosen. The SF mode requires one electrode to be brought to three different voltages at different parts of the sequence, which remains beyond the capability of fast high-voltage switches despite our best 


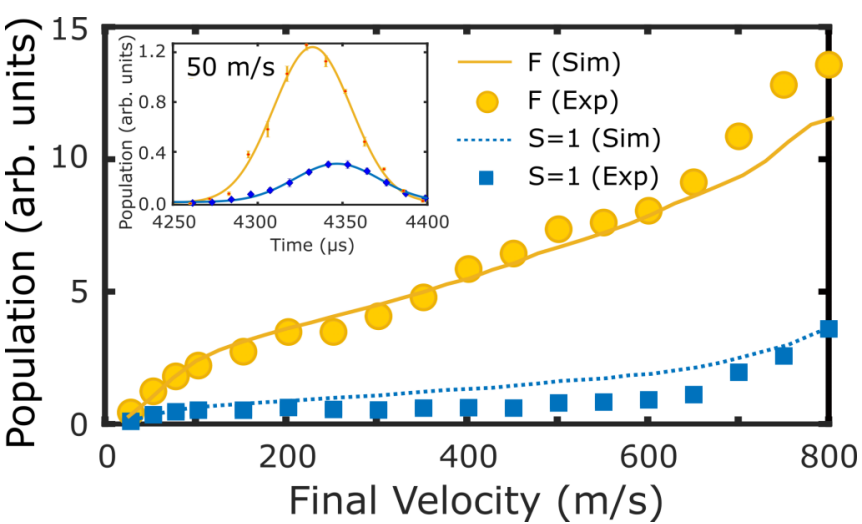

FIG. 3. The molecular signal and enhancement between the F mode and conventional $S=1$ deceleration over a range of final speeds. Data are collected with a beam of hydroxyl radicals expanded in neon at an initial speed of $825 \mathrm{~m} / \mathrm{s}$ and deceleration up to $200 \mathrm{~km} / \mathrm{s}^{2}$. The inset shows the time-of-flight signal from the valve pulse for $\mathrm{F}$ (orange) and $\mathrm{S}=1$ (blue) modes measured at the end of the decelerator when slowing to $50 \mathrm{~m} / \mathrm{s}$, demonstrating a factor-of- 4 improvement at trappable final speeds. Here the decelerator voltage is $12.5 \mathrm{kV}$.

efforts. Data are collected with a beam seeded in neon and an initial speed of $825 \mathrm{~m} / \mathrm{s}$, and run times ranging from 2 to 4 $\mathrm{ms}$ as the molecules are slowed to different final speeds. Pin spacing and most other device parameters are as previously reported $[11,40]$, but with increased length. In the F mode, signal decreases gradually and predictably with final speed, while in the $S=1$ mode signal declines rapidly with reduced final speed at first, but then plateaus once the improved focusing with stronger deceleration for this mode takes over. The result is that the $\mathrm{F}$ mode quadruples $\mathrm{S}=1$ at the lowest and highest final speeds, and improves by more than an order of magnitude in the central $400-500 \mathrm{~m} / \mathrm{s}$ range where $\mathrm{S}=1$ is at its worst. For low final velocities below $50 \mathrm{~m} / \mathrm{s}$ that are used for trap loading, separate studies will need to be performed to determine just how helpful these focusing modes prove. We anticipate that the improved uniformity of the phase space delivered by the F mode could simplify the notoriously challenging trap loading optimization process.

A particularly direct demonstration of the improved transverse focusing of the $\mathrm{F}$ mode results from varying decelerator voltage, as done for $S=1$ in Ref. [15] (Fig. 4). OH has a linear Stark shift in our field strengths, so adjusting the voltage linearly scales the potential it experiences. For operation modes with transverse focusing that is decoupled from the longitudinal, the voltage increase should only improve performance, deepening the traveling trap. Figure 4 shows the final population of molecules slowed using $\mathrm{S}=1$ and $\mathrm{F}$ modes to $50 \mathrm{~m} / \mathrm{s}$ under different decelerator voltages. At low enough voltages, the field between the pins is not sufficient to remove enough energy per stage, and molecules cease to be decelerated. As voltage increases, molecules slowed in the $\mathrm{S}=1$ mode do not need to approach the pins as closely, reducing the sampling of the interpin focusing field and worsening performance. Since the F mode separates transverse focusing from slowing, molecules experience greater transverse focusing at higher field strengths, giving rise to the observed

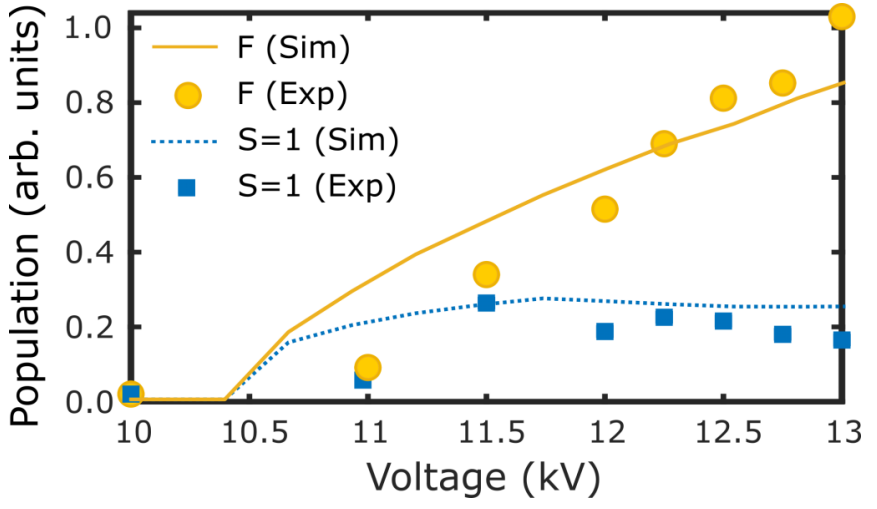

FIG. 4. Comparisons of decelerated populations between the F mode and $\mathrm{S}=1$ mode at different applied voltages with a final velocity of $50 \mathrm{~m} / \mathrm{s}$. The points represent experimental results, while the lines are calculated via Monte Carlo simulation. Instead of showing saturation behavior as $\mathrm{S}=1$, the decelerated population using the F mode increases with higher applied voltage.

approximately linear improvement above $11 \mathrm{kV}$. While we are currently limited to $13 \mathrm{kV}$ by the safety margins of our device, efficiency gains and greater phase-space acceptances should persist at even higher voltages, until the initially populated phase-space distribution becomes the limitation. At this point, skimmer cooling $[41,42]$ offers further benefits.

\section{CONCLUSION}

We introduce a deceleration strategy, with two accompanying modes of operation for the conventional pulsed decelerator. Significant improvements in overall performance are demonstrated. In contrast to deceleration in the $S=1$ mode, transverse focusing is directly applied by dedicated field distributions with much less dependence on the longitudinal coordinate, enabling further performance gains with increased voltage. The removal of this dependence also resolves openings in the traveling trap which previously resulted in significant losses. Less favorable molecules with smaller ratios of Stark shift to mass should benefit even more significantly than $\mathrm{OH}$, since they require longer travel times for deceleration. This opens up possibilities for successfully applying Stark deceleration to many new species, for example water and its isotopologs [43]. In addition to the two operation modes identified here, a whole class of deceleration modes incorporating new field distributions is ready for exploration.

\section{ACKNOWLEDGMENTS}

We acknowledge funding support for this work from NIST, ARO-MURI, and NSF Grant No. PHY-1734006. P.W. acknowledges support from the Polish Ministry of Science and Higher Education "Mobility Plus" Program. T.L. acknowledges support from the Alexander von Humboldt-Stiftung through a Feodor Lynen Fellowship.

\section{APPENDIX: TUNABLE FOCUSING DURATION}

In the main text, the focusing pulses introduced by $\mathrm{F}$ and SF operating modes are not specified more precisely than 
that they are applied when the molecule would otherwise pass between grounded electrodes, and the timing diagrams of Fig. 1 depict the focusing pulses as precisely symmetric about the grounded pin pair. Here we describe this much more fully. Unlike $S=1$ operation, where the timing of pulses is fully decided by a choice of desired final speed, the extra focusing pulses create a new timing degree of freedom which must be resolved. A highly similar challenge was tackled in Ref. [18], where extra switching events between the two field distributions used in $S=1$ were introduced, and a careful procedure was utilized to resolve the extra degrees of freedom while also optimizing relevant parameters. For the $\mathrm{F}$ mode, we do not find more than $10 \%$ gains in molecule number, either in simulation or experiment, by varying the focusing pulse length beyond the symmetric choice. For the
SF mode, we find that pulse length variations beyond the symmetric choice allow a direct tradeoff between longitudinal traveling well depth and transverse. The reason for this is that in the SF mode, the longitudinal potential energy (Fig. 1, bottom right) is large enough that applying it asymmetrically about the grounded pin pair leads to a relevant removal or addition of longitudinal energy. In simulation, we find that the optimum balance is to lengthen the pulse somewhat, and we incorporate this in the curves shown in Fig. 2 for SF. Specifically, for each deceleration, we optimize the SF focusing pulse length for phase-space volume and report the optimum in Fig. 2(a). The optimization also seems to maximize similarity between transverse and longitudinal traveling well depth [see Fig. 2(c)], a desirable characteristic for subsequent applications.
[1] S. Y. T. van de Meerakker, H. L. Bethlem, and G. Meijer, Nat. Phys. 4, 595 (2008)

[2] S. Y. T. van de Meerakker, H. L. Bethlem, N. Vanhaecke, and G. Meijer, Chem. Rev. 112, 4828 (2012).

[3] B. C. Sawyer, B. K. Stuhl, M. Yeo, T. V. Tscherbul, M. T. Hummon, Y. Xia, J. Kłos, D. Patterson, J. M. Doyle, and J. Ye, Phys. Chem. Chem. Phys. 13, 19059 (2011).

[4] M. Kirste, X. Wang, H. C. Schewe, G. Meijer, K. Liu, A. van der Avoird, L. M. C. Janssen, K. B. Gubbels, G. C. Groenenboom, and S. Y. T. van de Meerakker, Science 338, 1060 (2012).

[5] Z. Gao, T. Karman, S. N. Vogels, M. Besemer, A. van der Avoird, G. C. Groenenboom, and S. Y. T. van de Meerakker, Nat. Chem. 10, 469 (2018).

[6] J. Veldhoven, J. Küpper, H. L. Bethlem, B. Sartakov, A. J. A. Roij, and G. Meijer, Eur. Phys. J. D 31, 337 (2004).

[7] E. R. Hudson, H. J. Lewandowski, B. C. Sawyer, and J. Ye, Phys. Rev. Lett. 96, 143004 (2006).

[8] B. L. Lev, E. R. Meyer, E. R. Hudson, B. C. Sawyer, J. L. Bohn, and J. Ye, Phys. Rev. A 74, 061402(R) (2006).

[9] A. Fast, J. E. Furneaux, and S. A. Meek, Phys. Rev. A 98, 052511 (2018).

[10] H. Bethlem, F. Crompvoets, R. Jongma, S. van de Meerakker, and G. Meijer, Phys. Rev. A 65, 053416 (2002).

[11] B. C. Sawyer, B. L. Lev, E. R. Hudson, B. K. Stuhl, M. Lara, J. L. Bohn, and J. Ye, Phys. Rev. Lett. 98, 253002 (2007).

[12] B. C. Sawyer, B. K. Stuhl, D. Wang, M. Yeo, and J. Ye, Phys. Rev. Lett. 101, 203203 (2008).

[13] D. Reens, H. Wu, T. Langen, and J. Ye, Phys. Rev. A 96, 063420 (2017).

[14] S. Y. T. van de Meerakker, N. Vanhaecke, H. L. Bethlem, and G. Meijer, Phys. Rev. A 73, 023401 (2006).

[15] B. C. Sawyer, B. K. Stuhl, B. L. Lev, J. Ye, and E. R. Hudson, Eur. Phys. J. D 48, 197 (2008).

[16] S. Y. T. van de Meerakker, N. Vanhaecke, H. L. Bethlem, and G. Meijer, Phys. Rev. A 71, 053409 (2005).

[17] L. Scharfenberg, H. Haak, G. Meijer, and S. Y. T. van de Meerakker, Phys. Rev. A 79, 023410 (2009).

[18] D. Zhang, G. Meijer, and N. Vanhaecke, Phys. Rev. A 93, 023408 (2016).
[19] L. P. Parazzoli, N. Fitch, D. S. Lobser, and H. J. Lewandowski, New J. Phys. 11, 055031 (2009).

[20] S. Hou, S. Li, L. Deng, and J. Yin, J. Phys. B: At. Mol. Opt. Phys. 46, 045301 (2013).

[21] A. Osterwalder, S. A. Meek, G. Hammer, H. Haak, and G. Meijer, Phys. Rev. A 81, 051401(R) (2010).

[22] J. van den Berg, S. Mathavan, C. Meinema, J. Nauta, T. Nijbroek, K. Jungmann, H. Bethlem, and S. Hoekstra, J. Mol. Spectrosc. 300, 22 (2014).

[23] M. I. Fabrikant, T. Li, N. J. Fitch, N. Farrow, J. D. Weinstein, and H. J. Lewandowski, Phys. Rev. A 90, 033418 (2014).

[24] M. Quintero-Pérez, P. Jansen, T. E. Wall, J. E. van den Berg, S. Hoekstra, and H. L. Bethlem, Phys. Rev. Lett. 110, 133003 (2013).

[25] S. Hou, Q. Wang, L. Deng, and J. Yin, J. Phys. B: At. Mol. Opt. Phys. 49, 065301 (2016).

[26] Y. Shyur, J. A. Bossert, and H. J. Lewandowski, J. Phys. B: At. Mol. Opt. Phys. 51, 165101 (2018).

[27] N. Vanhaecke, U. Meier, M. Andrist, B. H. Meier, and F. Merkt, Phys. Rev. A 75, 031402(R) (2007).

[28] E. Narevicius, A. Libson, C. G. Parthey, I. Chavez, J. Narevicius, U. Even, and M. G. Raizen, Phys. Rev. Lett. 100, 093003 (2008).

[29] E. Lavert-Ofir, S. Gersten, A. B. Henson, I. Shani, L. David, J. Narevicius, and E. Narevicius, New J. Phys. 13, 103030 (2011).

[30] K. Dulitz, M. Motsch, N. Vanhaecke, and T. P. Softley, J. Chem. Phys. 140, 104201 (2014).

[31] Q. Wang, S. Hou, L. Xu, and J. Yin, Phys. Chem. Chem. Phys. 18, 5432 (2016).

[32] T. Cremers, S. Chefdeville, N. Janssen, E. Sweers, S. Koot, P. Claus, and S. Y. T. van de Meerakker, Phys. Rev. A 95, 043415 (2017).

[33] V. Plomp, Z. Gao, T. Cremers, and S. Y. T. van de Meerakker, Phys. Rev. A 99, 033417 (2019).

[34] P. Aggarwal, H. L. Bethlem, A. Borschevsky, M. Denis, K. Esajas, P. A. B. Haase, Y. Hao, S. Hoekstra, K. Jungmann, T. B. Meijknecht, M. C. Mooij, R. G. E. Timmermans, W. Ubachs, L. Willmann, and A. Zapara, Eur. Phys. J. D 72, 197 (2018). 
[35] For strong field seekers, see H. L. Bethlem, A. J. A. van Roij, R. T. Jongma, and G. Meijer, Phys. Rev. Lett. 88, 133003 (2002); M. R. Tarbutt, H. L. Bethlem, J. J. Hudson, V. L. Ryabov, V. A. Ryzhov, B. E. Sauer, G. Meijer, and E. A. Hinds, ibid. 92, 173002 (2004).

[36] H. L. Bethlem, G. Berden, A. J. A. van Roij, F. M. H. Crompvoets, and G. Meijer, Phys. Rev. Lett. 84, 5744 (2000).

[37] This is not highly apparent in Fig. 1, A and A', where a $45^{\circ}$ slicing plane is chosen for visual clarity. The defocusing is strongest in the plane including the decelerator axis that is also normal to the cylindrical axis of the grounded pins.
[38] S. Y. T. van de Meerakker, P. H. M. Smeets, N. Vanhaecke, R. T. Jongma, and G. Meijer, Phys. Rev. Lett. 94, 023004 (2005).

[39] U. Even, Adv. Chem. 2014, 636042 (2014).

[40] J. R. Bochinski, E. R. Hudson, H. J. Lewandowski, and J. Ye, Phys. Rev. A 70, 043410 (2004).

[41] Y. Segev, N. Bibelnik, N. Akerman, Y. Shagam, A. Luski, M. Karpov, J. Narevicius, and E. Narevicius, Sci. Adv. 3, e1602258 (2017).

[42] H. Wu, D. Reens, T. Langen, Y. Shagam, D. Fontecha, and J. Ye, Phys. Chem. Chem. Phys. 20, 11615 (2018).

[43] M. Motsch, L. D. van Buuren, C. Sommer, M. Zeppenfeld, G. Rempe, and P. W. H. Pinkse, Phys. Rev. A 79, 013405 (2009). 